\title{
Extensively Drug Resistance Process
}

National Cancer Institute

\section{Source}

National Cancer Institute. Extensively Drug Resistance Process. NCI Thesaurus. Code C102633.

Resistance to nearly all known drugs, particularly the most efficacious or non-first line drugs, used in the treatment of a particular disease. 